\title{
Development of Microelectromechanical Systems (MEMS) forceps for intraocular surgery
}

\author{
R B Bhisitkul, C G Keller
}

Br J Ophthalmol 2005;89:1586-1588. doi: 10.1136/bjo.2005.075853

\begin{abstract}
Aim: To develop silicon microforceps for intraocular surgery using Microelectromechanical Systems (MEMS) technology, the application of microchip fabrication techniques for the production of controllable three dimensional devices on the micrometre scale.

Methods: Prototype MEMS forceps were designed and manufactured for intraocular surgery. Scanning electron microscopy was used to evaluate device tip construction. Designs using both thermal expansion actuators and conventional mechanical activation were tested in human cadaver eyes and in vivo rabbit eyes to assess functionality in standard vitreoretinal surgery. Results: MEMS forceps were constructed with various tip designs ranging from $100 \mu \mathrm{m}$ to $2 \mathrm{~mm}$ in length. Scanning electron microscopy confirmed accurate construction of micro features such as forceps teeth as small as tens of micrometres. In surgical testing, the silicon forceps tips were effective in surgical manoeuvres, including grasping retinal membranes and excising tissue. The mechanical actuator design on a 20 gauge handle was more operational in the intraocular environment than the thermal expansion actuator design. While handheld operation was possible, the precision of the forceps was best exploited when mounted on a three axis micromanipulator. Conclusion: MEMS microforceps are feasible for conventional vitreoretinal surgery, and offer advances in terms of small scale, operating precision, and construction tolerance.
\end{abstract}

W hile vitreoretinal surgical instrumentation has undergone refinements over the past several decades, basic aspects of retinal instruments-materials, scale, and construction-have not departed in a fundamental way from established designs. The ability to achieve surgical objectives in ophthalmology is determined at least in part by the limits of instrumentation in terms of size and precision. Microelectromechanical Systems (MEMS) is a broad technology that utilises the materials and techniques of silicon microchip fabrication to create movable, controllable devices on the scale of micrometres to millimetres. ${ }^{1}{ }^{2}$ From commercial and industrial applications, increasing interest is directed towards biomedical applications of MEMS. ${ }^{3-6}$

Using MEMS technology, we have designed and manufactured micro-forceps prototypes for intraocular surgery, with instrument tips of single crystal silicon construction on the scale of $100 \mu \mathrm{m}$ and design features on the scale of $10 \mu \mathrm{m}$. Testing under standard vitreoretinal surgery conditions demonstrated their feasibility and functionality. To our knowledge this represents the first report of MEMS instruments for intraocular surgery.

\section{MATERIALS AND METHODS MEMS fabrication process}

Original designs for a variety of MEMS forceps were manufactured at the University of California Berkeley Microfabrication Laboratory. Forceps were constructed from two dimensional designs using boron doped silicon wafers (orientation (100), resistivity $0.02 \mathrm{ohm}-\mathrm{cm}$ ). Photoresist was spun onto wafers and patterned using a photomask, then hard baked at $125^{\circ} \mathrm{C}$. Deep etching was performed using the Bosch process in an STS plasma etcher. After cleaning wafers with acetone, piranha $\left(\mathrm{H}_{2} \mathrm{SO}_{4} / \mathrm{H}_{2} \mathrm{O}_{2}\right)$, and deionised (DI) water rinse, a $1 \mu \mathrm{m}$ thermal oxide coat was grown $\left(1100^{\circ} \mathrm{C}\right.$, $\mathrm{O}_{2}$, steam). Oxide was removed from the backside of the wafer using 5:1 buffered oxide etch (aqueous hydrofluoric acid (HF) with ammonium fluoride). A ring of oxide was preserved at the outer diameter of the wafer, to preserve the full wafer thickness around the edge to prevent it from becoming too fragile to handle. Wafers were placed in $25 \%$ tetramethyl ammonium hydroxide at $60^{\circ} \mathrm{C}$ until the etch front reached the bottom of the plasma etched pattern (where only oxide windows remain). Wafers were rinsed in DI water and all oxide was removed with $49 \% \mathrm{HF}$. A $1 \mu \mathrm{m}$ thick layer of wet thermal oxide was grown $\left(1100^{\circ} \mathrm{C}, \mathrm{O}_{2}\right.$, steam) to remove sharp corners and stress concentrations, then all oxide was removed with $49 \%$ HF. Finally, silicon parts were obtained, and for some designs assembled with epoxy to a forceps actuator shaft.

\section{Scanning electron microscopy}

Scanning electron microscopy (EM) was done with a Jeol 6400. Silicon specimens were mounted on standard aluminium scanning EM stubs using colloidal carbon paint. Gold coating was not necessary for visualisation as the silicon alone is sufficiently conductive. Micrographs were obtained and stored digitally.

\section{Forceps design and construction}

Two different actuator designs were developed to control forceps operation. The initial design was an electrically heated thermal expansion actuator, which incorporated tweezer tips and heat sink fins as a single piece (fig 1A). A manual potentiometer was used to apply current to rapidly heat and cool the semiconductive silicon of the thermal actuator, causing expansion and contraction in length, to open and close the tips via a lever linkage.

The second generation of forceps used a more conventional mechanical actuation (figs $1 \mathrm{~B}$ and 2). MEMS silicon forceps tips were joined to a 20 gauge stainless steel instrument shaft enclosing a spring loaded opening mechanism, which included a microcalibration system for fine adjustments of the forceps tip excursion. To maximise stability, the mechanical actuator itself is electrically activated, via wire connections to a control switch, much like the automated MPC scissor familiar to retinal surgeons. ${ }^{7}$

Several tip configurations were designed to be suitable for intraocular surgery. The stiffness of tips made for these forceps ranged from 1 nanonewton $/ \mu \mathrm{m}$ to 100 micronewtons $/ \mu \mathrm{m}$.

Abbreviations: $\mathrm{DI}$, deionised; $\mathrm{EM}$, electron microscopy; $\mathrm{HF}$, hydrofluoric acid; MEMS, Microelectromechanical Systems; MVR, microvitreoretinal 

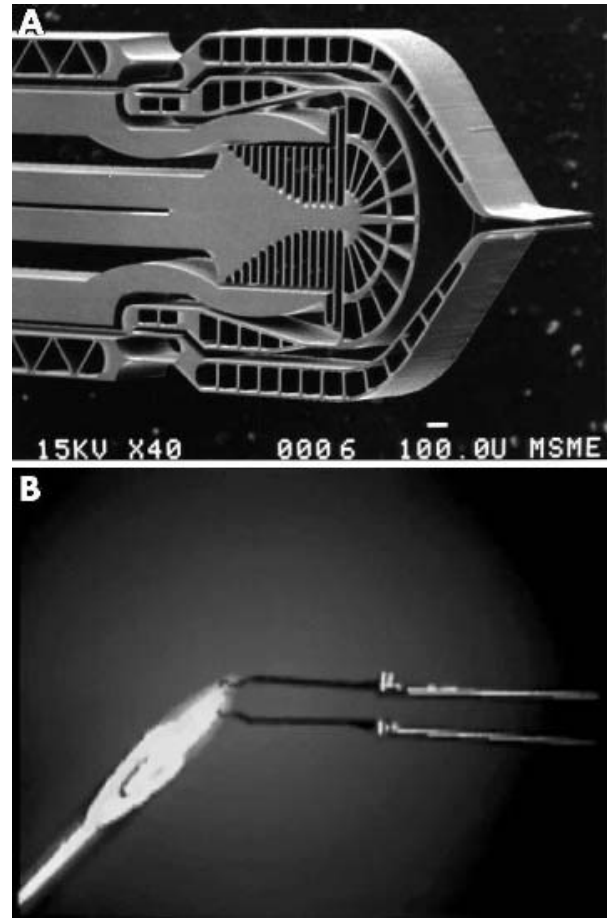

Figure 1 Prototype MEMS intraocular forceps. (A) Scanning electron micrograph of an early version of micro-tweezers with thermal expansion actuator. The device incorporates heat sink fins into the body of the tweezers; electric current actuates the tips by thermal expansion (calibration bar $=100 \mu \mathrm{m}$ ). (B) Later forceps designs employed a mechanical actuator system within a 20 gauge instrument shaft. (The forceps are shown with a 25 gauge needle in the background.)

\section{Intraocular surgery}

For surgical testing in human cadaver eyes, an eye cup was formed by excising the cornea, iris, and lens, then filling the vitreous cavity with balanced salt solution after vitrectomy. Surgery was done in an "open sky" fashion under the operating microscope (Zeiss Op-Mi6, Carl Zeiss, Germany). For in vivo surgical testing, standard three port 20 gauge vitrectomy (Storz Millenium, Rochester, NY, USA) was performed on adult New Zealand White rabbits (Charles River Laboratories Inc, Wilmington, MA, USA), anaesthetised with 3-5\% isoflourane mask inhalation (Baxter, Deerfield, IL, USA). Both lensectomy and lens sparing vitrectomies were done. The MEMS instruments were introduced into the eye through standard sclerotomies made with a 20 gauge microvitreoretinal (MVR) blade. At the completion of surgery animals were euthenised with intramuscular ketamine (30$50 \mathrm{mg} / \mathrm{kg}$, Fort Dodge Animal Health, Ft Dodge, IA, USA) and xylazine (5-10 mg/kg, Phoenix Pharmaceutical Inc, St Joseph, MO, USA), followed by intramuscular sodium pentobarbital (>150 mg/kg, Schering-Plough, Kenilworth, NJ, USA) and bilateral thoracotomy. All rabbit experiments were done in accordance with UCSF committee on animal research guidelines.

\section{RESULTS}

The instruments were manufactured at tip lengths from $100 \mu \mathrm{m}$ to $2 \mathrm{~mm}$; scanning EM confirmed the accurate construction of serrated teeth as small as $10 \mu \mathrm{m}$. Figure 2 shows a MEMS forceps with a serrated jaw design alongside a commercial stainless steel subretinal forceps, demonstrating the relative scale of the instrument tips as well as the high design tolerance made possible with MEMS technology.

Two different actuator systems were designed for the MEMS forceps. In the first prototypes, a thermal expansion
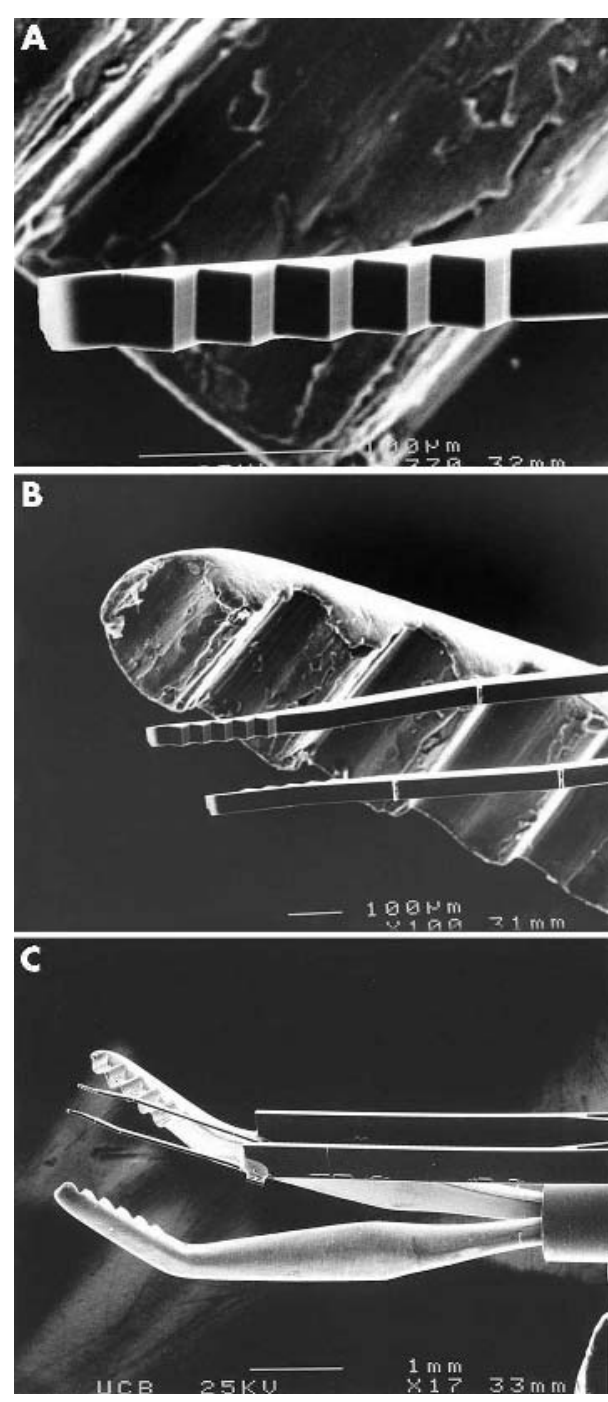

Figure 2 Scanning electron micrographs at different magnifications ( $A$, B) of mechanically actuated MEMS forceps with serrated jaws. In the background is a commercial stainless steel subretinal forceps. The shaft of the mechanical actuator to which the MEMS tips are glued is shown in (C). Calibration bars $=100 \mu \mathrm{m}$.

actuator adapted from engineering applications was redesigned for intraocular surgery. With this design, electrically heated beams (fig 1A), allow tip excursions as small as several micrometres. This actuator was usable in the eye only with an "open sky" approach, and frequently had thermal coagulation of materials on its surface after repeated current application which interfered with its mechanism.

Therefore, a redesign was made, based on a mechanical actuation system. A variety of MEMS designs were incorporated in a more conventional 20 gauge stainless steel spring loaded system. The circuit for its automated activation was enclosed within the shaft of the instrument and no thermal coagulation of surface materials was noted with repeated activation. For in vivo surgery in rabbit eyes with standard three port, 20 gauge vitrectomy, operation of the forceps was done in a conventional handheld fashion, and also by mounting the forceps on a Sutter Instruments three axis micromanipulator. Handheld operation was found to be feasible, but the micromanipulator provided greater stability and movement precision commensurate with the small scale of the instrument tips.

Surgical tests confirmed the viability of silicon as a material for intraocular instruments. The tensile properties 

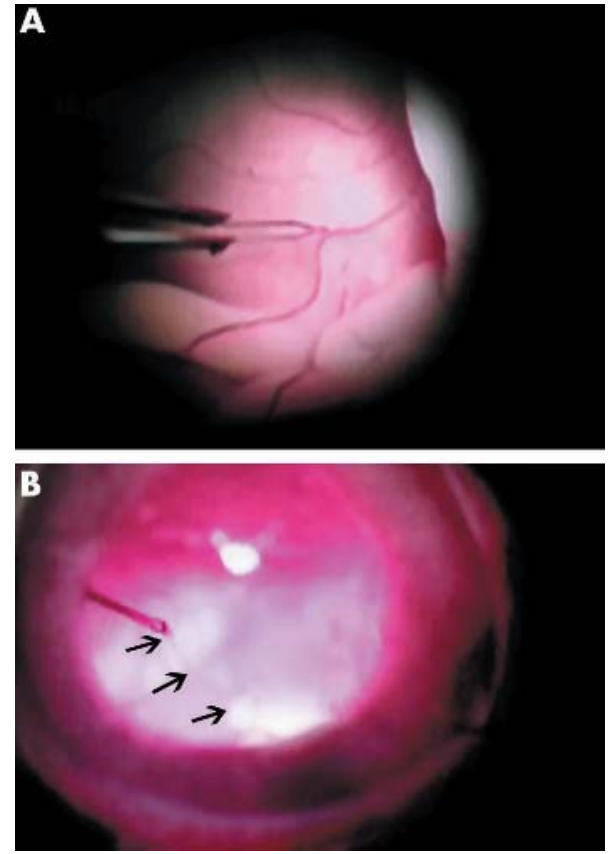

Figure 3 MEMS forceps in use during three port vitrectomy in the rabbit eye in vivo. (A) MEMS forceps grasping posterior hyaloid to manipulate detached retina. (B) MEMS forceps used to peel a fine preretinal membrane (arrows).

of silicon proved to be non-distensible and not plastically deformable under conditions of standard vitreoretinal surgery. Also, silicon was found to be antireflective with standard endoillumination, aiding visibility of the small tips. In surgical manoeuvres with serrated forceps (fig 3, and see video on $B J O$ website), we were able to firmly engage tissues and displace membranes without slippage. No breakage or fracture of the silicon tips was observed in multiple trials.

\section{DISCUSSION}

This project indicates the viability of MEMS forceps for intraocular surgery. The miniaturisation and construction tolerance of the MEMS forceps surpass that of commercially available stainless steel instruments. The tensile properties of silicon confer durability and function at small scales at which stainless steel would be plastically deformable. The material biocompatibility and sterilisability of MEMS devices and materials appear from initial studies to be satisfactory. ${ }^{5}$

MEMS fabricated instruments have potential applications in eye surgery. For example, the innovation of the 25 gauge vitrectomy system ${ }^{9}{ }^{10}$ has necessitated a rescaling of the full array of handheld vitreoretinal instruments, which MEMS instruments could complement and expand. Besides forceps, MEMS technology could be used to design membrane picks, blades, scissors, etc, on a scale much smaller than current products. The miniaturisation allowed by MEMS instruments could be used to advantage in advances such as nonvitrectomy retinal surgery. ${ }^{11}{ }^{12}$ Furthermore, since any two dimensional design template can be rapidly fabricated at a large scale, MEMS processes may facilitate advances in instrument design and even allow customisability for individual surgeons. Emerging research in biomedical MEMS includes drug delivery devices, micro-pumps, sensors, and retinal prostheses. ${ }^{13-16}$

Challenges remain with the development of MEMS intraocular instruments. In these tests, the initial prototype thermal expansion actuator was adapted from engineering and microscopy applications, but had disadvantages during eye surgery, exemplifying the disparities in transferring this technology from the laboratory setting to the intraocular environment. Improved systems for operative imaging, stabilisation, and micromanipulation may be required to realise the potential of MEMS microsurgery. In this project, we have not departed substantially from standard designs, but instead have advanced the scale and material of conventional forceps archetypes. However, MEMS technology may offer the capability to evolve instrument designs for surgical applications not foreseen presently.

\section{ACKNOWLEDGEMENTS}

This study was supported by That Man May See Foundation and by Research to Prevent Blindness.

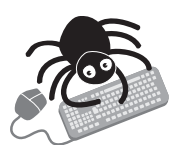

See video on www.bjophthalmol.com/supplemental

\section{Authors' affiliations}

R B Bhisitkul, Beckman Vision Center, Department of Ophthalmology, University of California San Francisco, San Francisco, CA, USA C G Keller, MEMS Precision Instruments, Richmond, CA, USA

Financial disclosure: CGK is founder of MEMS Precision Instruments; RBB has no financial interest.

Correspondence to: Robert B Bhisitkul, MD, PhD, Beckman Vision Center, Department of Ophthalmology, University of California San Francisco, 10 Koret Way, K301, San Francisco, CA 94143, USA; bhisit@itsa.ucsf.edu

Accepted for publication 1 August 2005

\section{REFERENCES}

1 Petersen KE. Silicon as a mechanical material. Proc IEEE 1982;70:420-57.

2 Wise KD, Najafi K. Microfabrication techniques for integrated sensors and microsystems. Science 1991;254:1335-42.

3 Roy S, Ferrara LS, Fleischman AJ, et al. Microelectromechanical Systems and neurosurgery: a new era in a new millennium. Neurosurgery $2001 ; 49: 779-98$.

4 Bashir R. BioMEMS: state-of-the-art in detection, opportunities and prospects. Adv Drug Deliv Rev 2004;56:1565-86.

5 Kotzar G, Freas M, Abel P, et al. Evaluation of MEMS materials of construction for implantable medical devices. Biomaterials 2002;23:2737-50.

6 Polla DL, Erdman AG, Robbins WP, et al. Microdevices in medicine. Ann Rev Biomed Eng 2000;2:551-76.

7 Machemer R, Parel JM, Hickingbotham D, et al. Membrane peeler cutter: automated vitreous scissors and hooked needle. Arch Ophthalmol 1981;99:152-3.

8 Weisenberg BA, Mooradian DL. Hemocompatibility of materials used in microelectromechanical systems: Platelet adhesion and morphology in vitro. $J$ Biomed Mater Res 2002;60:283-91.

9 Fujii GY, De Juan E Jr, Humayun MS, et al. A new 25-gauge instrument system for transconjunctival sutureless vitrectomy surgery. Ophthalmology 2002; 109:1807-12.

10 Fujii GY, De Juan E Jr, Humayun MS, et al. Initial experience using the transconjunctival sutureless vitrectomy system for vitreoretinal surgery. Ophthalmology 2002; 109:1814-20.

11 Saito Y, Lewis JM, Park I, et al. Non-vitrectomizing vitreous surgery: a strategy to prevent postoperative nuclear sclerosis. Ophthalmology 1999; 106:1541-5.

12 Sawa M, Saito Y, Hayashi A, et al. Assessment of nuclear sclerosis after nonvitrectomizing vitreous surgery. Am J Ophthalmol 2001;132:356-62.

13 Liepmann D, Pisano AP, Sage B. Microelectromechanical systems technology to deliver insulin. Diabetes Technol Ther 1999;1:469-76.

14 Polla DI, Erdmann AG, Robbins WP, et al. Microdevices in medicine. Annu Rev Biomed Eng 2000;2:551-76.

15 Li Y, Shawgo RS, Tyler B, et al. In vivo release from a drug delivery MEMS device. J Control Release 2004;100:211-19.

16 Zhou DD, Greenberg RJ. Microsensors and microbiosensors for retinal implants. Front Biosci 2005;10:166-79. 